Canadian

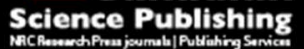

Canadian Journal of Civil Engineering Revue canadienne de génie civil

\title{
British Columbia Smart Infrastructure Monitoring System (BCSIMS)
}

\begin{tabular}{|c|c|}
\hline Journal: & Canadian Journal of Civil Engineering \\
\hline Manuscript ID & cjce-2016-0577.R2 \\
\hline Manuscript Type: & Article \\
\hline Date Submitted by the Author: & 01-Apr-2017 \\
\hline Complete List of Authors: & $\begin{array}{l}\text { KAYA, YAVUZ; University of British Columbia, Department of Civl } \\
\text { Engineering } \\
\text { Ventura, C.E.; Dept. of Civil Engineering } \\
\text { Huffman, Sharlie; B.C. Ministry of Transportation } \\
\text { Turek, Martin; University of British Columbia, Department of Civil } \\
\text { Engineering }\end{array}$ \\
\hline $\begin{array}{r}\text { Is the invited manuscript for } \\
\text { consideration in a Special } \\
\text { Issue? : }\end{array}$ & N/A \\
\hline Keyword: & $\begin{array}{l}\text { Strong Motion Network, Seismic Structural Health Monitoring, Shake-map, } \\
\text { Earthquake Notification, Real-time data processing }\end{array}$ \\
\hline
\end{tabular}




\section{British Columbia Smart Infrastructure Monitoring System (BCSIMS)}

\section{Yavuz Kaya, Carlos Ventura, Sharlie Huffman, Martin Turek}

Yavuz Kaya - Corresponding author: Research Engineer, Department of Civil Engineering, Faculty of Applied Science, University of British Columbia, BC, Vancouver, 6250 applied Science Lane, V6T 1Z4, Canada, Phone: +1 778-874-0243, Yavuz.Kaya@gov.bc.ca

Carlos Ventura: Professor, Department of Civil Engineering, University of British Columbia, BC, Vancouver, 6250 Applied Science Lane, V6T 1Z4, Canada, ventura@,civil.ubc.ca

Martin Turek: Research Engineer, Department of Civil Engineering, University of British Columbia, BC, Vancouver, 6250 Applied Science Lane, V6T 1Z4, Canada, meturek@mail.ubc.ca

Sharlie Huffman: Bridge Engineer, Huffman Engineering Ltd., 2180 Pentland Road, Victoria, BC, Canada, V8S 2W6, sharlieh@telus.net 


\begin{abstract}
This paper describes the architecture of a comprehensive seismic monitoring system developed in 2009 by the British Columbia Ministry of Transportation and Infrastructure (MoT) and the University of British Columbia, Canada. The main purpose of the BCSIMS project is to instrument and monitor key structures to provide confirmation of their seismic capacity, assist in focusing retrofit efforts, detect structural damage, and provide rapid damage assessment of those structures, following a seismic event. The automatic creation of shake-maps following an earthquake provides information for the MoT's non-instrumented bridges. The public notification systems and web pages have been developed to display real-time seismic data both from the Strong Motion Network and Seismic Structural Health Monitoring Network. Tools and methods have been developed to help transform the current practice of inspecting and evaluating structures to a more rational and effective one that uses the up-to-date sensing technology with fast and efficient techniques.
\end{abstract}

Keywords: Seismic Structural Health Monitoring, Strong Motion Network, Shake-map, Earthquake Notification, Real-time data processing 


\section{INTRODUCTION}

Southwest coast of British Columbia (BC), including Vancouver and the densely populated Lower Mainland, is located over an active subduction zone, which results in one of the most seismically active regions of Canada (Rogers 1998). This zone, called the Cascadia subduction zone, is capable of producing large, up to magnitude 9.0, earthquakes (Goldfinger et al. 2012). The last mega-quake in this region was $\sim 300$ years ago (Goldfinger et al. 2012) and evidence suggests that strain has been accumulating in the subduction boundary, off the west coast of Vancouver Island (Clague et al. 1992 and Gragert et al. 1994). Analysis of contemporary crustal deformation proves the same strain accumulation in the subduction boundary (Gragert et al. 1994; Dragert et al. 1994; Dragert and Hyndman 1995). Even though Vancouver has not yet experienced a large damaging earthquake, the paleo seismic evidence on the Fraser delta, south of Vancouver, confirms that large earthquakes have occurred in prehistoric time (Clague et al. 1992). Ongoing occurrence of earthquakes, which are large enough to cause damage to structures, is a reminder of the fact that the southwest cost of BC is still a seismically active area. Other smaller, but still potentially damaging, earthquakes may also occur in the overlapping crust and in the subduction slab. Such seismic activity, as a result, presents hazard to the area and risk to the civil engineering structures built in those areas.

In order to help mitigate this seismic risk, the Geologic Survey of Canada (GSC) through the Pacific Geoscience Centre (PGC) has maintained an urban Strong Motion Network (SMN) in BC since 2003. As part of this network, the GSC developed a strong motion Internet Accelerograph (IA) network, which is permanently connected to the Internet and records ground vibration data continuously. The instrument continuously computes a set of Strong Motion (SM) parameters, which characterize the intensity of shaking, and actively reports those values to the GSC's and the University of British Columbia's (UBC) data centres via 4 relays whenever ground shaking 
exceeds predefined threshold levels. Over the last several years, the British Columbia Ministry of Transportation and Infrastructure (MoT) has been working with the PGC to expand the range of the network outside of urban centres and increase the number of stations in the network to the current 162 strong motion stations.

The MoT is responsible for $400 \mathrm{~km}$ of provincial disaster response routes in $\mathrm{BC}$. The loss of any portion of these routes after an earthquake could significantly impact emergency response efforts and negatively affect public well-being. The MoT, moreover, maintains over 2500 bridges in the highest seismic zones, many of which are vulnerable to extensive damage in even a moderate quake and potential collapse in a major earthquake. The loss of the use of several structures would not only have immediate impact on public well-being and the ability of emergency vehicles to respond effectively, but would also cripple the economic recovery of the region. The better the information on these areas where structures and facilities are most vulnerable, the better the planning and the preparation can be done. By identifying those structures and facilities most susceptible to seismic forces through automatically generated shake-maps, decision-makers can do effective risk management. Fast and accurate field intelligence immediately following an earthquake can ensure the most effective deployment of vital services and mitigate damage to the built environment.

In a parallel effort, the MoT has also been instrumenting bridges and tunnels in collaboration with the Earthquake Engineering Research Facility (EERF) at the UBC since the late 1990's. The primary purpose of these legacy systems was to capture the ground motion input and its effect on structures in the event of an earthquake. Building on these collaborations, the MoT and the UBC recently embarked on a program called the British Columbia Smart Infrastructure Monitoring System (BCSIMS), which integrates data from the instrumented structures (currently sixteen in total) and the SMN. The system organizes and processes real-time data in an efficient manner and 
delivers results and related reports to predefined recipients such as bridge inspectors at the MoT. This instrumentation program provides immediate notification after an event and incorporates remote Structural Health Monitoring (SHM) system. The goals of the system are: 1) to provide a real-time seismic structural response system to enable rapid deployment and prioritized inspections of the MoT's structures; 2) to develop and implement a structural health monitoring program to address the need for safe and cost-effective operation of structures in $\mathrm{BC}$; and 3 ) to provide a real-time working platform ( $\underline{\text { www.bcsims.ca) }}$ that can integrate many aspects of seismicity in $\mathrm{BC}$.

The implementation of BCSIMS transforms the current practice of inspecting and evaluating all structures after an earthquake to a more rational and effective one that makes effective use of state-of-the-art sensing technology with fast and efficient techniques for data analysis and interpretation; therefore, the inspections can then be focused and prioritized to maximize the effective use of the scarce resources.

\section{BCSIMS SYSTEM ARCHITECTURE}

The development of the BCSIMS architecture has been a collaborative process between the UBC and MoT that started in early 2009. Figure 1 shows an overview of the BCSIMS architecture, and it consists of several sub-systems: hardware and software, data acquisition system, data storage and processing tools, network communications, etc. In general, the BCSIMS network involves two main components: the SMN and the seismic SHM network. They are discussed in detail in the following subsections.

\subsection{Strong Motion Network}

\subsubsection{Internet Accelerometers (IA) Network}

The SMN, developed and currently maintained by the GSC and the MoT, consists of 170 Internet Accelerometers (IA) stations (Rosenberger et al. 2004), which are deployed across the province 
as shown in Figure 2. The IA stations are designed to detect and measure an earthquake in realtime, and send the recorded event data along with calculated seismic parameters over the Internet to a central location. This procedure promotes the idea of real-time response. The IA station has a digital recorder built internally that allows at least three days of raw data to be stored locally in a ring buffer. The IA stations are connected to the BCSIMS network via four relay serves, which are located at the UBC in Vancouver; the PGC in Sidney on the Vancouver Island; Kamloops in BC; and in Ottawa: this increases the robustness of the entire SM network.

The measured vibration data in the IA stations is filtered in real-time two times in parallel: 1-10 $\mathrm{Hz}$ band-pass and $0.1 \mathrm{~Hz}$ high-pass filters. The high-pass filtered data is utilized to calculate the important strong motion parameters in real-time such as the Peak Ground Acceleration (PGA), Peak Ground Velocity (PGV), Peak Ground Displacement (PGD) and various spectral intensity scales such as Katayama's Spectral Intensity (kSI) (Katayama et al. 1998). The band-pass filtered data, on the other hand, is used to calculate the ratio of the Short-time average to Long-time average (STA-LTA). A smart algorithm embedded in the IA station detects the early onset of an earthquake. The algorithm is based on the combination of the threshold values for these seismic parameters. When a set of triggered parameters are exceeded, the sensor logs the earthquake records as an event file and later begins processing it after the earthquake is over. Event files are permanently stored on the sensor. After the analysis is done, the raw data along with the calculated seismic parameters are synchronized to the global database via one of the relay servers. During this process, two types of string messages are sent out via relays to predefined recipients. The first message, which is sent out right after the sensor is triggered, contains the name of the sensor location and the date $\&$ time of the event, while the second message contains the same information as the first message and the calculated seismic parameters. The second message is relayed after the instrument is de-triggered. One of the recipients of these two messages is the main server located 
at the UBC. After receiving the second string message, the main server at UBC establishes a connection to the sensor through one of relay servers, downloads and processes the event file, and permanently stores it in a global SQL database.

The earthquake information (e.g. magnitude, location, hypo-central depth, date \& time, etc.) is read in real-time from online feed (USGS) and is permanently stored in the global database. Upon the registry of an earthquake in the global database at the UBC, two windows services are initiated. The first windows service sends out an earthquake notification email to predefined registered users of the BCSIMS network. There are currently 4 types of users: administrator, scientist, engineer, and public: the amount of information accessible on the webpage (www.bcsims.ca) depends on user type. The second windows service compares the date \& time information of the earthquakes to that of the event files in the global database. Shake-maps will be created for an earthquake if the following criterions are met:

- The epicentre of the earthquake is less than $200 \mathrm{~km}$ from the nearest IA station

- $\quad$ The magnitude of the earthquake is bigger than 3

- $\quad$ At least one IA station is triggered

These criterions are defined as default values in the system, but the system administrator can modify them at any time in order to effectively manage the BCSIMS network. A recent seismic event in British Columbia is used as an illustration of how the system performs. An earthquake with a magnitude of 4.8 (USGS) occurred near Sidney Island in BC (48.6038 Latitude and 123.3068 Longitude) on Wednesday 30th, 2015 at 07:39 AM UTC time. Fifty strong motion stations (IA stations) were triggered due to this shaking, and the earthquake was felt across the Lower Mainland, BC. The locations of the triggered stations are depicted in Figure 3, and the maximum horizontal acceleration of $0.04 \mathrm{~g}$ was recorded at the Brentwood Bay station on Vancouver Island, which is $11.47 \mathrm{~km}$ away from the epicenter of the earthquake. The magnitude 
of this earthquake is bigger than 3, more than one IA station is triggered, and the epicenter of the earthquake is less than $200 \mathrm{~km}$ from the nearest IA station; therefore, the BCSIMS system automatically generated a shake-map, which can be seen in Figure 4.

\subsubsection{Shake-maps}

These shake-maps are used by federal, provincial, and local organizations, both public and private, for post-earthquake response and recovery as well as for preparedness exercises and disaster planning. An application has been developed using Matlab to produce shake-maps for urban and regional scales. The core of the shake-map algorithm can be described in four steps as: 1) correction of the values of the PGA to rock site condition; 2) estimation of PGA amplitudes at virtual stations of generated grid using bias-corrected attenuation relationships (Boore and Atkinson 2008); and 3) numerical interpolation between grid points; and 4) amplification of the PGA values to original site condition. Peak horizontal accelerations at each IA station are used in the calculation of the PGA, but the peak values of the vertical components are not used because they are on average lower than the horizontal amplitudes. Ground Motion Prediction Equations (GMPE) (Boore and Atkinson 2008; Atkinson 2005; Atkinson and Boore 2011) are used to estimate the peak horizontal amplitudes in between IA stations. The Instrumental Intensity (II) shake-map is based on a combined regression of PGA and PGV versus observed intensity for significant earthquakes (Wald et al. 1999). Shake-maps are generated automatically following moderate and large earthquakes to represent the distribution of strong ground motion and shaking intensity in the region.

Shake-maps generated on the BCSIMS website can be viewed with different superimposed layers, such as bridges, buildings, or schools layer. This enables emergency responders and maintenance personnel to quickly assess the shaking intensity across the urban areas and at the 
location of critical infrastructure. It also allows these agencies to prioritize and maximize the effective use of their scarce resources available.

\subsubsection{Strong Motion Event Report}

Upon creating the shake-maps, a strong motion event report is automatically generated at the main server and e-mailed predefined subscribers lists, such as bridge inspection engineers of the MoT. The report includes the snapshot of the earthquake including typical parameters such as location, magnitude, depth, etc. It also lists the strong motion stations that are triggered by that earthquake and peak responses. The data recorded by each triggered station is automatically processed, and the results are included in this report. The processed data includes recorded acceleration, calculated velocity and displacement time histories as well as the acceleration response spectrum and the smoothed Fourier Amplitude Spectrum (Kaya and Safak 2014).

\subsubsection{BCSIMS Web Interface}

The www.bcsims.ca website is the gateway for user interaction and operational management of the BCSIMS network. Figure 2 shows a screen shot of the most recent version of the homepage of the website. The amount of information accessible on the webpage depends on user type. Public users have access to the strong motion network data and the shake-maps generated after an event, whereas authorized users are allowed to access further processed information such as automatically generated Strong Motion Event Reports and Structural Event Reports. The structure stations and the SMN are displayed as different icons on a digital geomap: the circles represent the SMN stations (Figure 2) and the squares are structural stations (Figure 7). The interactive map allows zooming in/out to focus on a particular station or area. By clicking on a station on the map, additional metadata is provided such as geographical location, picture of the station, and live links to webcams if available. A link in the metadata allows users to view the event data recorded by that station. This includes recorded raw acceleration, calculated velocity 
and displacement, Fourier amplitude spectrum, and the acceleration response spectrum. An option to view or download all of the recorded data is available in the website for advanced users to further investigate the recorded event data. Such event data is very useful for those bridges that are non-instrumented and located near an IA station. They could further be used to quickly assess the shaking intensity at or near a non-instrumented bridge.

The SM stations are connected to the BCSIMS network in real-time, and the home page of the website shows the location of the SM stations as blue circles. The size and colour of the circles change in real-time based on the reported PGA and kSI values at that SM station. In other words, all of the activities (e.g., seismic, impact, collision, etc.) of SM stations in the SMN are monitored and displayed in real-time using a coloured-circle on the geo map as shown in Figure 2. The size of the circle indicates the maximum measured amplitude of the recorded PGA within predefined time period, and the color depicts the maximum calculated $\mathrm{kSI}$ value. This feature allows the user to immediately assess the shaking intensity across the SMN in real-time before a shake-map is generated for the earthquake.

The user can further navigate through the menus on the website to view the earthquake activity in the west cost of Canada and USA. The epicenters are shown by a coloured-star, as shown in Figure 5, where the size of the star indicates the moment magnitude of the earthquake.

\subsubsection{Earthquake Simulation}

The earthquake simulation tool enables users either to simulate an earthquake on the website or to view the results of an already simulated earthquake. A simulation can be done with different earthquake magnitude, epicenter location, and earthquake type (e.g., subduction or crustal earthquake). The simulation is depicted by two growing yellow and red circles as shown in Figure 6. They indicate the $\mathrm{p}$ - and the s-waves propagations. The user can control the velocity of these waves as well as the epicentral location of the earthquake. Before the start of a situation, the 
colour of all of the IA stations on the map is blue by default indicating that none of them are triggered yet. Once the user starts the simulation, these two circles start growing on the map, and the colour of the IA stations will first change from blue to green in real-time. This means that the yellow circle ( $p$-wave) reached the IA station, and as result of that, the p-wave triggered that IA station. When the red circle (s-wave) reaches to the IA station, the colour of that station will change to red: the IA station started recording the vibration data. The simulation stops when the swave reaches the user defined distance from the epicenter. After the simulation is over, the final size and the colour of the IA stations on the map indicate the expected PGA and the kSI value for each station, respectively. The expected PGA is calculated based on the GMPE proposed by Boore at al. 2008; Atkinson 2005; and Atkinson and Boore 2011.

Simulated shake-maps provide useful information to emergency responders, bridge maintenance engineers, and other public and governmental agencies. The user can get an appreciation of the expected shaking intensity across urban areas and at the location of critical infrastructures before an actual earthquake occurs in that area.

\subsection{Structural Health Monitoring (SHM)}

The practical significance of seismic SHM cannot be overstated. After a significant earthquake, for example, it is very common to see a large number of slight-to-moderately damaged bridges, along with the heavily damaged or undamaged structures. The damaged structures typically require detailed and time consuming inspections to decide whether the damage is structural or not, and if the damage is of structural nature, then decide if it is safe to re-occupy them. This process can result in heavy financial losses for the owners of such structure. The SHM systems in the BCSIMS network provide the means to help make such decisions faster and with more confidence. 
In parallel to recent developments in sensor and recording technologies, most of the structures in the BCSIMS network are now installed with SHM systems, which involves continuous monitoring of the dynamic characteristics of structures by digital instruments (e.g., accelerometers, displacement transducers, environmental sensors, etc.). The main objective of the SHM part of the BCSIMS is to track the changes in the dynamic characteristics of the monitored structure in order to detect and locate damage, and to make fast decisions on the safety level of the structure, and the actions that need to be taken.

Figure 7 depicts the locations of the bridge structures in the BCSIMS network, and Table 1 shows a list of the structures that have seismic SHM system installed and are currently being monitored in real-time. A sensor in Table 1 means a single channel of a data acquisition system that measures a structure's physical response quantity such as acceleration or displacement. The instrumentation system installed at each structure is capable of remote configuration and can automatically upload data to multiple remote servers via the Internet. Each instrumented structure in the BCSIMS has a Data Acquisition (DAQ) unit to retrieve and store data from each sensor on the structure. The type and the number of the sensor used in a seismic SHM system usually depends on the dynamic characteristics of the structure and the intentions of the seismic SHM system installed. For instance, a wind sensor located at the top of the towers of the Port Mann Bridge helps to better understand the relationship between the wind speed \& its direction and the amplitude of the acceleration on the deck and towers.

The collected data from each structure is archived and processed in real-time on the Data Processing Server (Figure1). The data processing includes drift analysis, modal identification, and the calculation of the important statistics of each data channel such as mean, root-meansquare (RMS) and standard deviation and so on. Finite element model updating, the damage detection, and structural event reports are other important features that have been developed and 
implemented in BCSIMS. The results of all of these analyses are permanently stored on the global database in the main server. The entire SHM network is currently run at UBC. An additional back-up server is being planned to set up at Ministry's main data base center. As a physical complement to the web based monitoring network, a control room has been established at UBC. As seen in Figure 8, the control room has been developed as a situation-room with all the necessary skilled human resources and enabling technologies available.

\subsubsection{Data archiving}

In order to streamline the data transmission process and overcome the proprietary data format of different hardware suppliers, UBC has developed its own data archiving standards and protocols. The collected data at the DAQ is streamed via Internet to a data processing server at UBC where they are stored in 5-minute lengths of Virtual Input File (VIF). The VIF file is a binary file that contains raw data from all of the sensors for each monitored structure. The length of the VIF file is user adjustable, and it is set to 5-minute by default because this length enables data processing server to keep up with the post processing of the data from all of the structures simultaneously. As soon as a new VIF file becomes available in the data processing server, it is further compressed so as to minimize the disk space. The compressed data is then stored in a two-week length ring buffer in a designated folder on the data processing server. The length of the ring buffer is scalable based on the available disk space on the server. The advantage of this approach is that it enables to achieve consistency and platform-neutrality across all data acquisition systems and hardware platforms, thereby simplifying the downstream processing. Moreover, this unique data archiving technique not only enables to select the most suitable hardware for a specific bridge (e.g., for technical performance or cost effectiveness), but also offers flexibility in replacing (e.g., defective) sensors in the future. 
A certain amount of raw data (about 30 minutes) from each structure is permanently stored on the data processing server every day. The length of such data is structure specific and determined based on the dynamic characteristics of each structure. The collection of such data from each structure is called "scheduled-measurement" and it is used to test the new tools and techniques that are continuously developed as part of the BCSIMS project. The current emphasis is on developing damage detection and localizations tools.

\subsubsection{Statistics}

The one of the key requirements in real-time seismic SHM is that recording, processing, and analysis of the data should all be done in real-time. The seismic SHM system can track slow changes in the characteristics of the structure, such as those due to aging, change of usage, traffic patterns, and other environmental factors (e.g., temperature, wind, rain, etc.). The change of modal properties of the structure especially due to environmental conditions can be larger than the change due to the damage; therefore, the effect of environmental conditions on modal properties of structure must be accounted for as they can completely mask the change of modal properties caused by actual damage (Kaya et al. 2013). Due to the continuous recording in the SHM, the statistical characteristics of the structural changes and their correlation with the factors that might cause such changes are established using statistical parameters like mean, standard deviation, root-mean-square, etc. These parameters help to better understand the dynamic behaviour of the bridge under different loading conditions, such as seasonal temperature change and daily traffic loads on a bridge, etc. This also enables building up a statistical history of the correlation between the dynamic response of the bridge and the environmental factors so that the effect of environment can be adequately accounted for in the recorded data. This information is very helpful to determine whether the identified change in a structural parameter actually represents damage or not (Kaya et al. 2013; Peeters and Roeck 2001). 


\subsubsection{Drift analysis}

Structural damage at bridge piers are often directly related to displacement demands. It is usually controlled in many seismic bridge codes by imposing displacement (or drift) limits on these structural members. The drift at a bridge pier is defined as the relative displacement of the pier top with respect to its base; therefore, in order to calculate the drift, the displacements at these two locations need to be measured or estimated from recorded acceleration data at the top and the base of the pier. It is common practice to calculate displacements from acceleration data by double integration of the recorded accelerations. However, the integration operation on raw data significantly increases the noise amplitudes in the integrated signal and can lead to misleading results (Kaya and Safak 2014). The drift calculation tool developed for BCSIMS minimizes such noise increase. The tool band-pass filters the recorded data around each resonant frequency of the bridge pier, and later combines the results to obtain the total displacement or drift for each pier. The response of a bridge pier at resonant frequency has much higher signal-to-noise ratio; therefore, the noise influence during the integration and differentiation is minimum.

The administrator of the BCSIMS network specifies the drift pairs for each of the instrumented piers on a bridge, and the system computes the drift from the integrated displacement values. The peak values during an event are stored in the global database. Any drift value exceeding a predefined threshold value may be indicative of possible damage in the structure, and if this is the case, an e-mail notification is sent out to a predefined list of users. The drift thresholds for each pier are determined based on the Canadian Highway Bridge Design Code requirements and results from detailed nonlinear finite element model analyses.

\subsubsection{Modal Identification}

The SHM system in the BCSIMS estimates the modal parameters of the structure (e.g., modal frequencies, modal damping ratios, and mode shapes) from output-only data (e.g., recorded 
bridge vibration data only) using the Stochastic Subspace Identification (SSI) (Overschee and Moor 2011; Overschee and Moor 1991; Peeters and Roeck 1999; Ljung 1999) method. The SSI method was introduced in 1991, and the first application to modal identification problems was reported in 1995 (Peeters et al. 1995; Peeters and Roeck 1999). The SSI has many advantages over other modal identification methods, the most important of which, among many others, is that the method can be fully automated so that no human interaction is necessary.

The modal parameters provide very important information about the dynamic characteristics of the structure. As soon as new vibration data from any structure in the BCSIMS network becomes available in the data processing server, the modal properties of that structure are automatically estimated in near real-time using the SSI method, and the results are stored in the main server (Figure1). The estimated modal properties of each structure are then incorporated in a control chart, which enables to track them against time. The identified modal properties are further used for finite element model updating and damage detection.

\subsubsection{Finite element model updating and damage detection}

An initial Finite Element (FE) model is created for each instrumented structure, and a two-step FE model updating is implemented. In the first step, the FE model updating is done based on an on-site detailed Ambient Vibration Test (AVT). This updated FE model represents the undamaged structure. The updated model can be used for several different purposes, such as detailed seismic assessment of the bridge. In the second step, the FE model is further updated automatically using the identified modal properties of the bridge after a severe event (e.g., a seismic event) where the modal characteristics of the FE model are calibrated/updated by ensuring that the updated FE model better reflects the measured/recorded data than the initial FE model. The updated FE model is then used to detect the damage on the structure by comparing the updated FE model to the undamaged FE model in the first step. This method is based on the 
fundamental idea that damage will cause detectable changes in modal properties of the structure (Fan and Qiao 2011; Doebling et al. 1996). This process has been fully automated in the BCSIM network.

A damage detection algorithm, which is based on the state-space formulation and its residuals (Dohler et al 2013; Basseville et al 2004), has been implemented and improved to detect the damage on structures. The method uses the covariance of the recorded vibrations and does not require the FE model of the structure. One desirable feature for this method is its ability to detect damage even in the presence of noise and common environmental effects such as wind speed and its direction, temperature, and traffic load changes. It is a robust method and less affected by changes due to ambient conditions. The method makes use of a data clustering in order to increase the speed of the algorithm and the accuracy of the damage detection. A baseline model is determined and continuously updated using the measurements from the undamaged structure, the length of which differs for each structure. A statistical-based threshold value is automatically calculated using the baseline model, but such threshold value is also user selectable. As soon as a new set of measurement data becomes available in the data processing server, a damage index is calculated for that set of data and stored permanently in the global database. If the seismic SHM on the structure is triggered for any reason, such damage index is calculated automatically. Calculated damage indexes are then posted in a control chart against a user-definable set of damage indicators (Dohler et al. 2013).

The damage detection algorithms implemented in the BCSIMS system provide reliable intelligence and run simultaneously in near real-time for each instrumented structure, and a notification is issued via e-mail to predefined subscribers list when damage is detected.

\subsubsection{Structural event report}


As soon as an event is detected in BCSIMS, it initiates an event recording on the Data Processing Server, and the recorded event data is permanently stored in a designated secure directory on the Data Processing Server after the seismic event is over. The Structural Event Report, which is issued approximately 5-miutes after the seismic event is over, will be automatically generated, emailed to predefined subscribers list, and published on the BCSISM website. This report provides key information on the status of each bridge after an earthquake. One report will be issued for each structure. This event report may also be issued for several reasons in addition to seismic events such as impact, over loading, wind, ship collision with bridge, or simply for a scheduled health assessment of the structure.

This structural event Report provides bridge inspection engineers with the shaking amplitude at the various locations of the bridge as well as the amplitude of the ground shaking. These recorded amplitudes are automatically compared with the code-specified design values (or user selectable thresholds) in the report. Any recorded value that exceeds the design values is clearly indicated in the report by graphs and figures, and recommendations are also given in the report with regards to the actions to be taken.

\section{SUMMARY AND CONCLUSION}

The British Columbia Ministry of Transportation and the University of British Columbia, in collaboration with other organizations, have embarked on a program, which is called the British Columbia Smart Infrastructure Monitoring System (BCSIMS). The system integrates data both from instrumented bridge structures and strong motion networks. It effectively organizes and processes these data in an efficient manner and deliver the collected information along with processed data to the appropriate parties such as bridge engineers and bridge area managers in the Ministry of Transportation. The processed data involves statistical analysis, results of model identification and calibrated Finite element models of structures. The strong motion event report 
and the structural event report provide the MoT and other public and government agencies with additional level information so that they can manage their emergency response resources effectively in the event of an earthquake.

Even though the tools and techniques that have been developed in the BCSIMS have been tested by several small to moderate earthquakes since its first installation in 2009 , there is only one earthquake that triggered the entire system on Wednesday 30th, 2015, and that particular earthquake was used as an example in this study to validate the BCSIMS system. The results show that the BCSIMS system can successfully respond to earthquakes as it was designed for and provides the required information that the bridge engineers and bridge area managers in the MoT need in order to make decisions and respond to emergencies in an efficient manner.

\section{ACKNOWLEDGEMENT}

The BCSIMS project has fully funded by the Ministry of Transportation (MoT) BC since 2009. Some of the tools and techniques in BCSIMS project have been jointly developed and programed by Structural Vibration Solutions (SVS) of Aalborg in Denmark and by the French Institute for Research in Computer Science and Automation (INRIA) in Rennece in France. The authors are greatly acknowledge the MoT, SVS, and INRIA for their contributions.

\section{ACRONYMS}

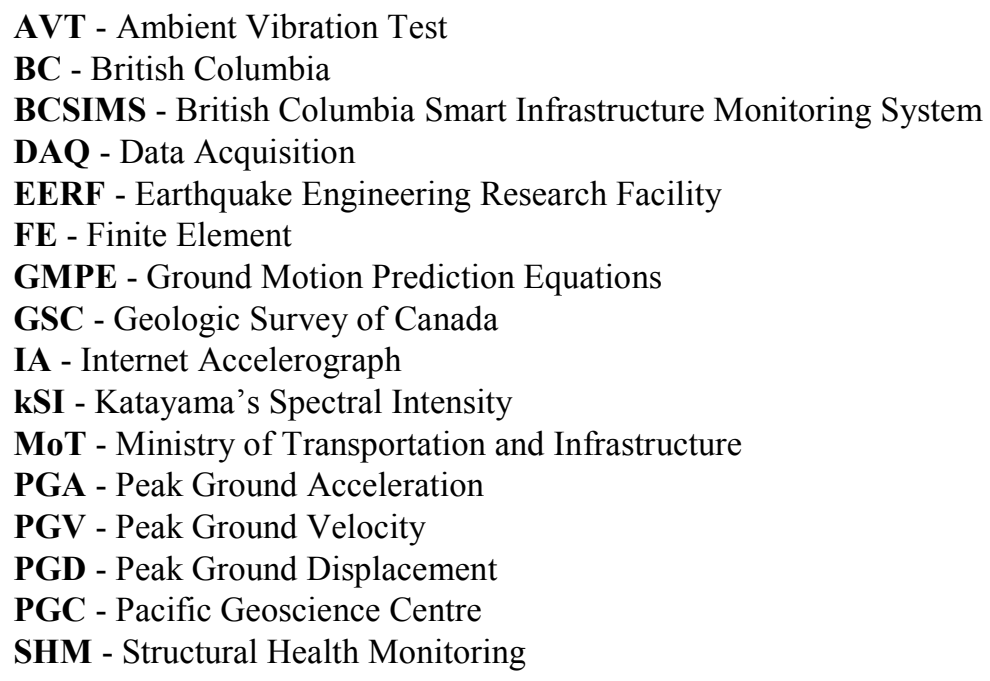


STA-LTA - Short-time average to Long-time average

SMN - Strong Motion Network

SM - Strong Motion

RMS - Root-Mean-Square

UBC - University of British Columbia

VIF - Virtual Input File

\section{REFERENCES}

Atkinson, G.M. 2005. Ground Motions for Earthquakes in Southwestern British Columbia and Northwestren Washington: Crustal, In-Slab, and Offshore Events, Bulletin of Seismological Society of America, v. 95, no. 3, p. 1027-1044

Atkinson, G.M., and Boore, D.M. 2011. Modifications to Existing Ground-Motion Prediction Equations in Light of New Data, Bulletin of Seismological Society of America, v. 101, no. 3, p. 1121-1135

Basseville, M., Mevel, L. and Goursat, M. 2004. Statistical model-based damage detection and localization: subspace-based residual and damage-to-noise sensitivity ratios, Journal of Sound and vibration, v 275, p 769-794

Boore, D.M. and Atkinson, G.M., 2008. Ground-Motion Prediction Equations for the Average Horizontal Component of PGA, PGV, and 5\%-Damped PSA at Spectral Periods between $0.01 \mathrm{~s}$ and $10.0 \mathrm{~s}$, Earthquake Spectra, Volume 24, No. 1, pages 99-138, February 2008

Clague, J.J., Naesgaard, E., and Sy, A., 1992. Liquefaction features on the Fraser delta: evidence for prehistoric earthquakes, Canadian Journal of Earth Science, v.29, pp.1734-1745.

Doebling, S.W., Farrar, C.R., Prime, M.B., and Shevitz, D.W. 1996. Damage identification and health monitoring of structural and mechanical systems from changes in their vibration characteristics: a literature review, Report LA13070-MS. Los Alamos National Laboratory, Los Alamos, NM

Dohler, M., Mevel, L. and Hille, F. 2013. Subspace-based damage detection under changes in the ambient excitation statistics, Mechanical Systems and Signal Processing, v45, p 207-227

Dragert, H., Hyndman, R. D., Rogers, C., and Wank K., 1994. Current deformation and the width of the seismogenic zone of the northern Cascadia subduction thrust, Journal of Geophysical Research, v.99, p. 653-668.

Dragert H., Hyndman R. D., 1995. Continuous GPS Monitoring of Strain in the northern Cascadia subduction zone, Geophysical Research Letters, v.22, pp. 755-758.

Fan, W. and Qiao, P. 2011. Vibration-based damage identification methods: a review and comparative study. Structural Health Monitoring 10(1):83-111

Goldfinger C., Nelson C. H., Morey A. E., Johnson J. E., Patton J. R., Karabanov E., Getierrez-Pastor J., Eriksson A. T., Eulalia G., Dunhills G., Enkins R., Dallimore A., Vallier T. 2012. Turbidite event history: Methods and implications for Holocene paleoseismicity of the Cascadia Subduction Zone, United States Geological Survey Professional Paper

Gragert H., Hyndman R. D., Rogers g. C., Wank K., 1994. Current deformation and the width of the seismogenic zone of the northern Cascadia subduction thrust, Journal of Geophysical Research, v.99, p. 653-668

Hastie T., Tibshirani R. and Friedman J. 2011. The Elements of Statistical Learning: Data Mining, Inference, and Prediction, Springer Series in Statistics

Hoehn, L. and Niven, I., 1985. Averages on the Move, Mathematics Magazine v. 58, No 3, p. 151-156

Karim, K.R. and Yamazaki, F., 2002. Correlation of JMA instrumental seismic intensity with strong motion parameters, Earthquake Engineering and Structural Dynamics, 2002; 31:1191-1212 
Katayama, T., Sato, N. and Saito, K. 1998. SI-Sensor for the Identification of Destructive Ground Motion, Proc. Ninth World Conference of Earthquake Engineering, Tokyo-Kyoto, VII p. 667-672

Kaya, Y. and Safak, E., 2014. Real-time analysis and interpretation of continuous data from structural health monitoring (SHM) systems, Bulletin of Earthquake Engineering, DOI 10.1007/s10518-014-9642-9

Kaya, Y., Turek, M., Ventura, C., 2013. Temperature and Traffic Load Effects on Modal Frequency for a Permanently Monitored Bridge, Conference Proceedings of the Society for Experimental Mechanics Series 38, DOI 10.1007/978-1-4614-6519-5_6

Ljung L., System Identification: Theory for the User (2nd Edition), Prentice Hall PTR, 1999.

MATLAB Commercial Integrating Technical Computing Program, The MathWorks Inc., Natick, MA, USA, 2000, www.mathworks.com

Overschee, P.V. and Moor, B.D., 2011. Subspace Identification for Linear Systems: Theory, Implementation and Applications, Kluwer Academic Publishers, Dordrecht, the Netherlands.

Overschee, P.V. and Moor, B.D.1991. Subspace algorithms for the stochastic identification problem. In Proceedings of the 30th IEEE Conference on Decision and Control, Brighton, UK; $321\{1326$.

Peeters, B., Roeck, G.D., Pollet, T., and Schueremans, L. 1995. Stochastic subspace techniques applied to parameter identification of civil engineering structures. In Proceedings of New Advances in Modal Synthesis of Large Structures: Nonlinear, Damped and Nondeterministic Cases, Lyon, France, p. 151-162.

Peeters, B. and Roeck, G.D., 1999. Reference-based stochastic subspace identification for output-only modal analysis. Mechanical Systems and Signal Processing 1999; 13(6): p. 855-878.

Peeters, B. and Roeck, G.D. 2011. One-year monitoring of the Z24-Bridge: environmental effects versus damage events, Earthquake Engineering and Structural Dynamics, 2001; 30:149-171

Rogers, G. C., 1998. Earthquakes and Earthquake Hazard in the Vancouver area; in Geology and Natural Hazards of the Fraser River Delta, British Columbia, Geological Survey of Canada, Bulletin 525, pp. 17-25

Rosenberger, A., Beverley, K., and Rogers, G., 2004. The new strong motion seismic network in southern British Columbia, CANADA, 13th World Conference on Earthquake Engineering Vancouver, B.C., Canada. Paper No. 3373

USGS, United States Geological Survey, http://earthquake.usgs.gov/earthquakes/feed/v1.0/

Wald, D.J., Quitoriano, V., Heaton, T.H., Kanamori, H. 1999. Relationship between Peak Ground Acceleration, Peak Ground Velocity, and Modified Mercalli Intensity in California, Earthquake Spectra, v. 15, no. 3 
Figure 1: The architecture of the BCSIMS that consists of several servers, hardware \& software, data acquisition system, data storage and processing tools, and network communications. All of them are connected to central servers located at UBC via Internet.

Figure 2: Strong Motion (SM) network: the homepage of the BCSIMS network. The SM network encompasses 162 Strong Motion stations deployed across the province of British Columbia and sixteen instrumented structures (i.e., bridge and tunnel). Each circle on the geo-map indicates one SM station, and its colour and size change in real-time. The size of the circle indicates the recorded PGA, and the colour indicates the kSI (Katayama et al. 1998) calculated.

Figure 3: Locations of the triggered stations due to Mw4.8 earthquake occurred near Sidney Island in BC. The location of the epicenter is indicated by a red star on the map. A total of 50 IA stations are triggered, and the circles on the map indicate the locations of these stations. The size of the circle is proportional to the recorded peak ground acceleration, and the color of the circle shows the calculated kSI (Katayama et al. 1998).

Figure 4: Calculated shake-map for the Mw4.8 earthquake occurred on December $30^{\text {th }}, 2015$ at 07:39AM (UTC). Maximum horizontal acceleration of $0.04 \mathrm{~g}$ was recorded at the Brentwood Bay station on Vancouver Island (11.47km away from the epicenter).

Figure 5: Recorded earthquake activities from December $1^{\text {st }}, 2015$ to November $30^{\text {th }}, 2016$ in the west cost of Canada and the USA. The epicenters of the earthquakes are indicated by colouredstars whose size is proportional to the magnitude of the earthquake.

Figure 6: Earthquake simulation tool on the BCSIMS website enables users to simulate p- and swaves of an earthquake. The two-growing yellow and red circles indicate the theoretical p- and swaves propagation. As these waves propagate, the colour of the SM stations will change from blue to green and red: the p- and s-waves triggered the IA station. After the simulation is over, the final size and the colour of the IA stations indicate the expected PGA and kSI (Katayama et al. 1998).

Figure 7: The locations of bridges owned by the Ministry of Transportation in British Columbia. The numbers in the square indicates number of bridges located under that square, and there are 2594 bridges in total and 14 of them have seismic SHM monitoring system installed (Table 1).

Figure 8: Monitoring control room at UBC, (a) the main server and data processing server in the control room, (b) the situation-room established in the control room. 
Table 1: List of tunnel, bridges, and buildings that are instrumented and currently being monitored in real-time in the BCSIMS network

\begin{tabular}{cccccc}
\hline No & Structure Name & $\begin{array}{c}\text { Total Length } \\
(\mathbf{m})\end{array}$ & Inst. Year & $\begin{array}{c}\text { Number of } \\
\text { channel }\end{array}$ & Type of Sensor \\
\hline 1 & French Creek (FC) & 200 & 1997 & 12 & A \\
2 & George Massey Tunnel (GMT) & 660 & 1996 & 11 & A P \\
3 & Queensborough (QB) & 914 & 1996 & 12 & A P \\
4 & Ironworkers Memorial Second & 1290 & 2011 & 122 & A S W T \\
5 & Narrows Crossing (IMSNC) & & & & \\
6 & Pitt River (PR) & 380 & 2009 & 46 & A W \\
7 & William R. Bennett (WRB) & 1077 & 2008 & 12 & A \\
8 & Portage Creek Bridge (PCB) & 129 & 1983 & 41 & A S \\
9 & Port Mann (PM) & 850 & 2013 & 336 & A W D T H P \\
10 & Gaglardi Way Underpass (GWU) & 65 & 2013 & 22 & A T H T H \\
11 & Kensington Avenue Underpass (KAU) & 75 & 2013 & 30 & A T H \\
12 & Fraser Heights - Wetlands (FHW) & 476 & 2013 & 20 & A T H \\
13 & 8264 BNSP Sunbury & 68 & 2014 & 36 & A H W D \\
14 & 8270 BNSF Viaduct East Mill Access & 195 & 2014 & 84 & A H W D \\
15 & 8313 Hwy-17 Deltaport & 133 & 2014 & 36 & A H W D \\
& Earthquake Engineering Research & - & 2013 & 16 & A \\
\hline
\end{tabular}

A: Acceleration, P: Piezometer, S: Strain gauge, W: Wind, T: Temperature, D: Displacement, H: Humidity 


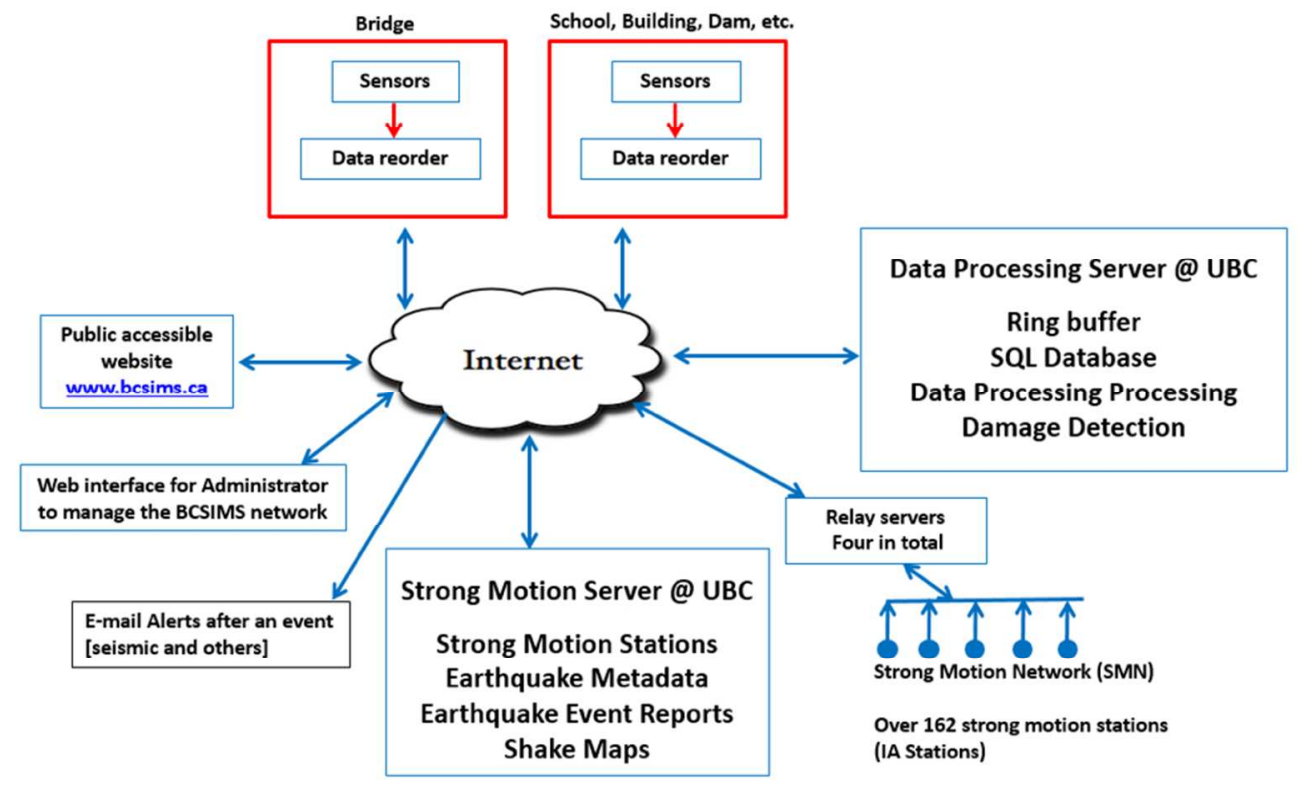

The architecture of the BCSIMS that consists of several servers, hardware \& software, data acquisition system, data storage and processing tools, and network communications. All of them are connected to central servers located at UBC via Internet.

$82 \times 50 \mathrm{~mm}(300 \times 300 \mathrm{DPI})$ 


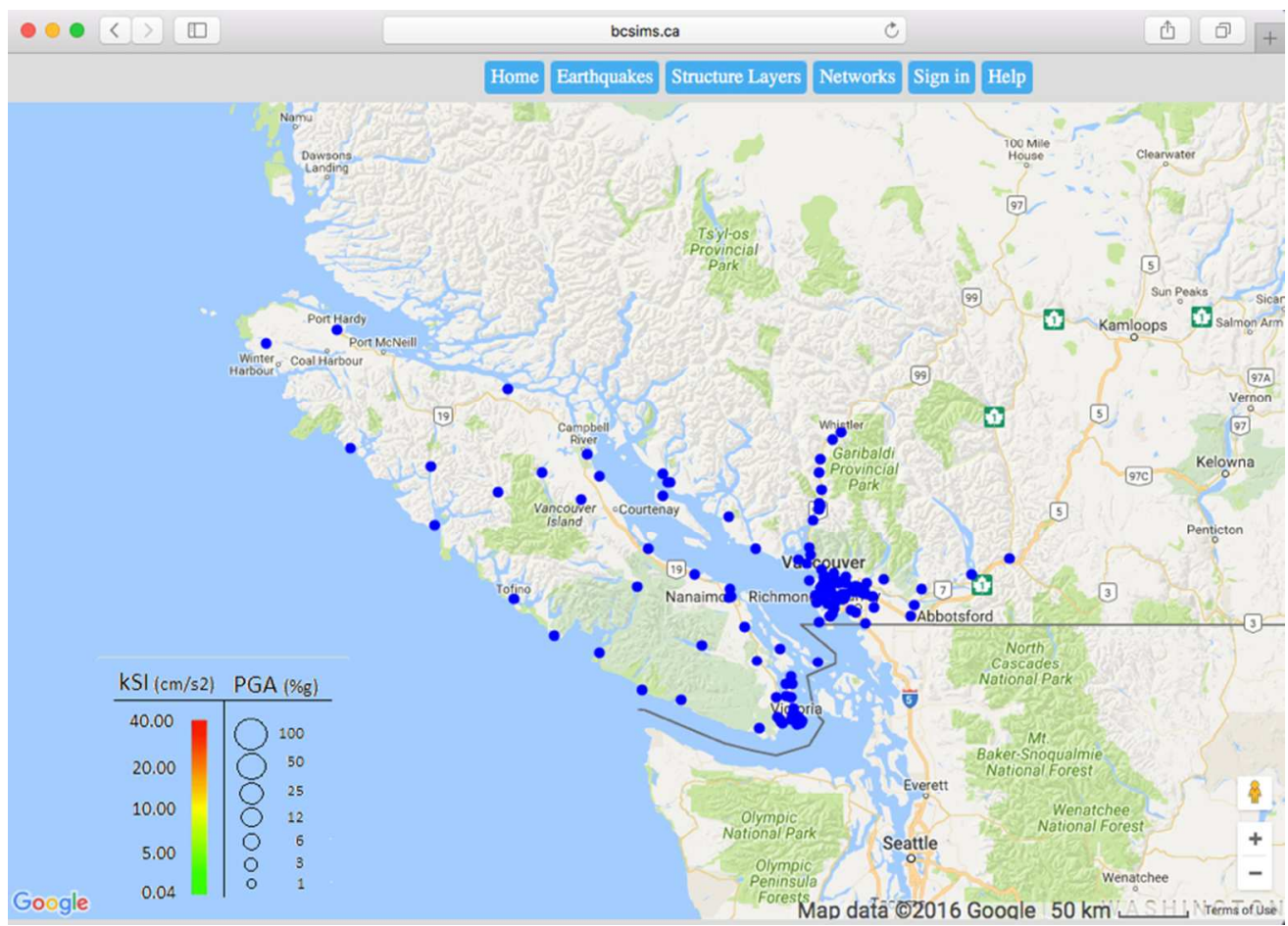

Strong Motion (SM) network: the homepage of the BCSIMS network. The SM network encompasses 162 Strong Motion stations deployed across the province of British Columbia and sixteen instrumented structures (i.e., bridge and tunnel). Each circle on the geo-map indicates one SM station, and its colour and size change in real-time. The size of the circle indicates the recorded PGA, and the colour indicates the kSI (Katayama et al. 1998) calculated.

$82 \times 59 m m(300 \times 300$ DPI $)$ 


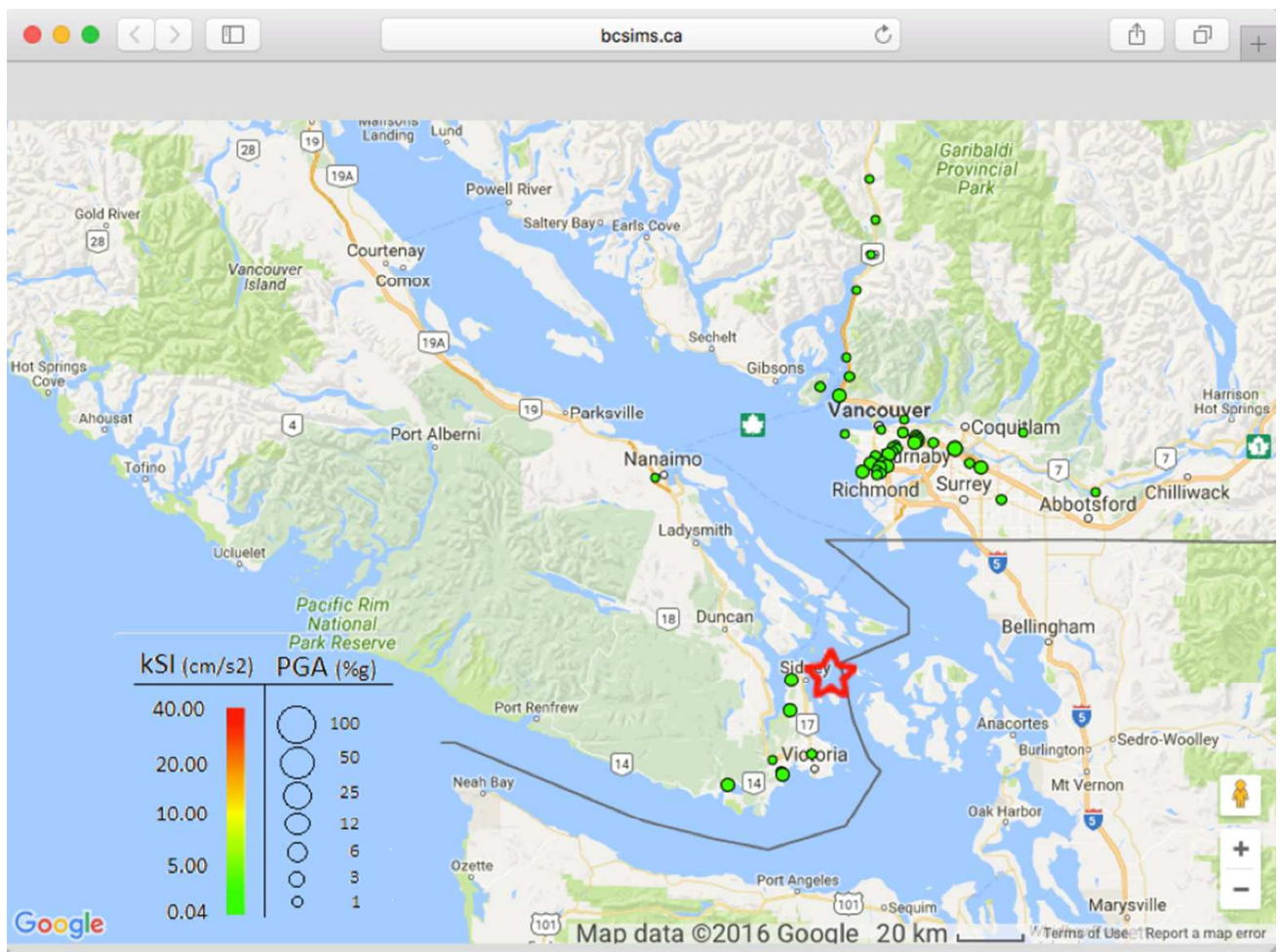

Locations of the triggered stations due to Mw4.8 earthquake occurred near Sidney Island in BC. The location of the epicenter is indicated by a red star on the map. A total of 50 IA stations are triggered, and the circles on the map indicate the locations of these stations. The size of the circle is proportional to the recorded peak ground acceleration, and the color of the circle shows the calculated kSI (Katayama et al. 1998).

$82 \times 61 \mathrm{~mm}(300 \times 300 \mathrm{DPI})$ 


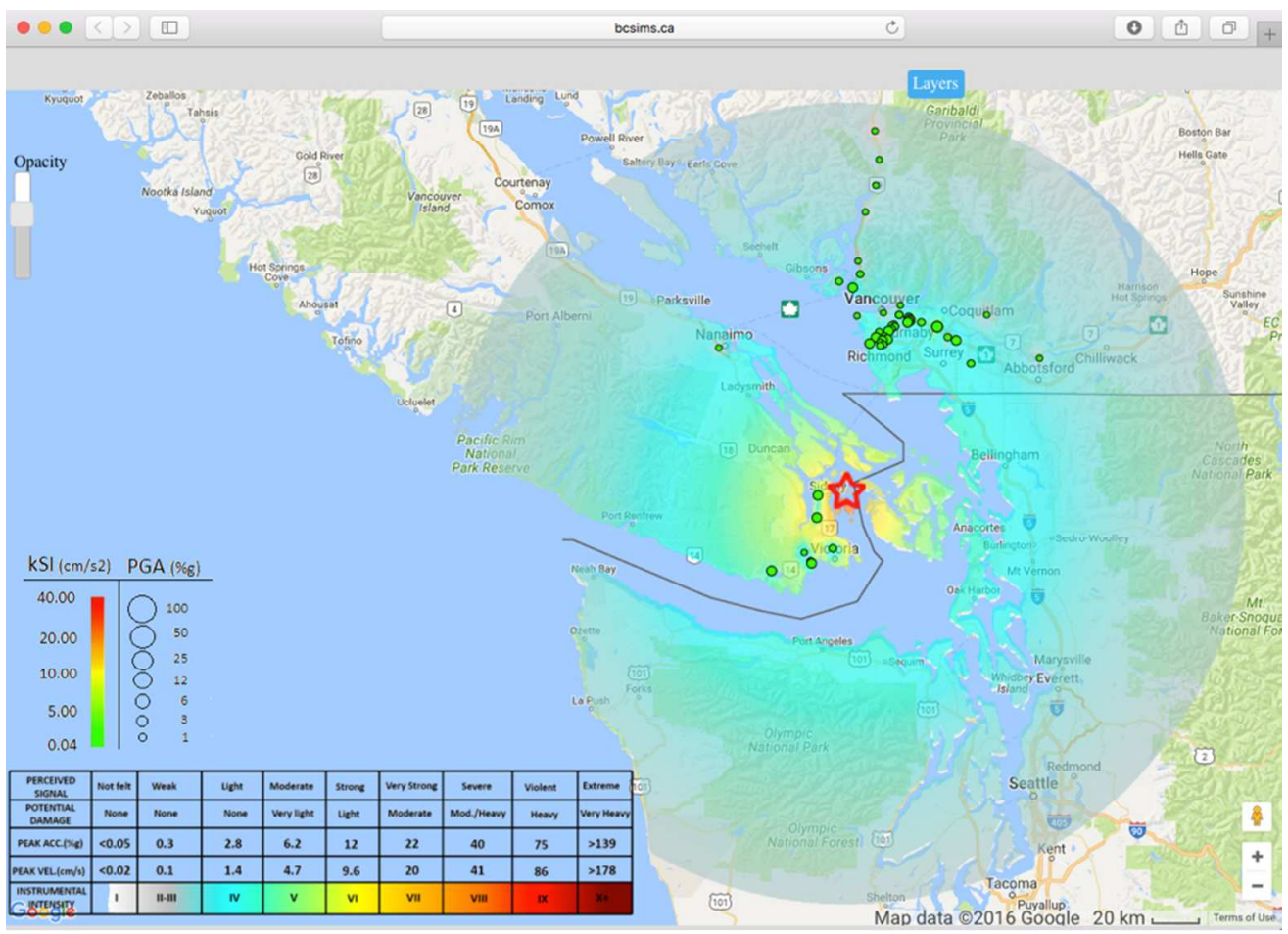

Calculated shake-map for the Mw4.8 earthquake occurred on December 30th, 2015 at 07:39AM (UTC). Maximum horizontal acceleration of $0.04 \mathrm{~g}$ was recorded at the Brentwood Bay station on Vancouver Island $(11.47 \mathrm{~km}$ away from the epicenter).

$82 \times 59 \mathrm{~mm}(300 \times 300 \mathrm{DPI})$ 


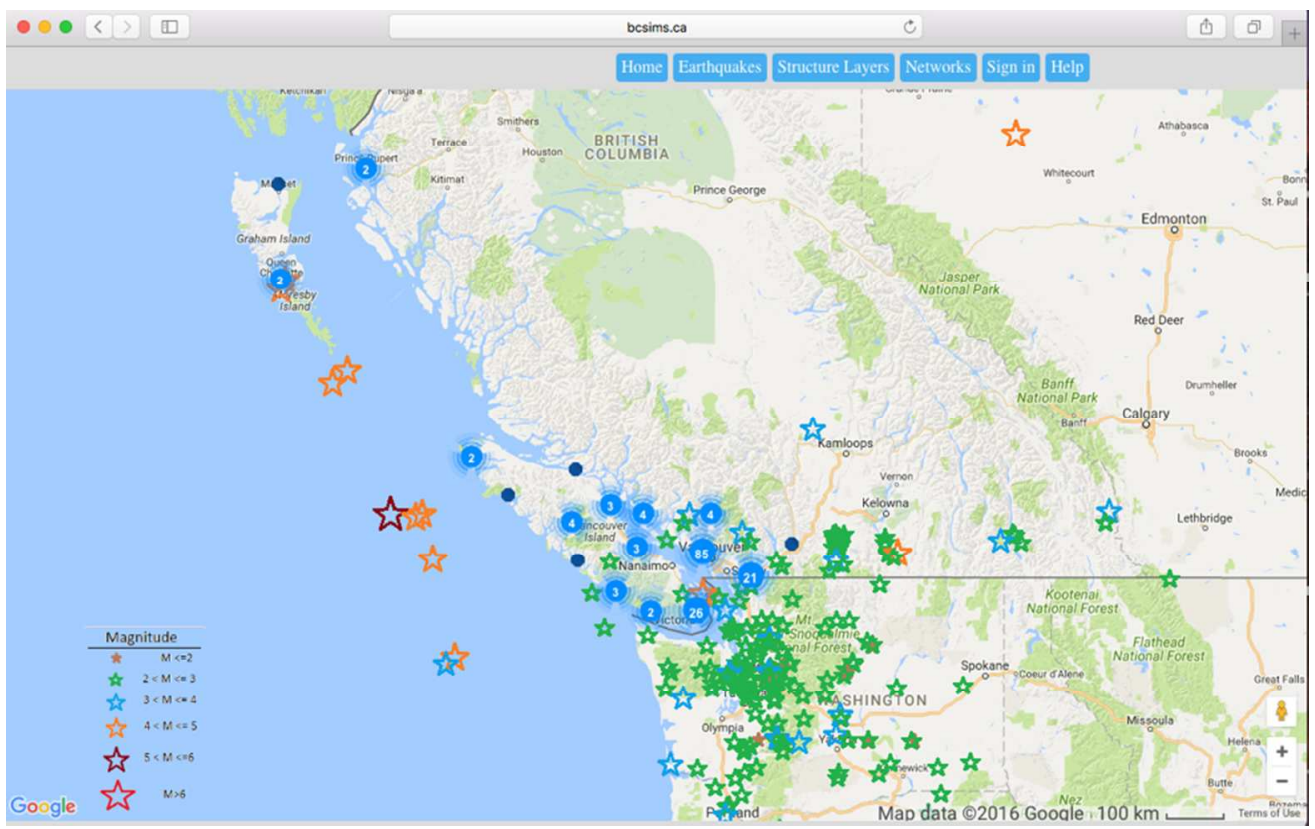

Recorded earthquake activities from December 1st, 2015 to November 30th, 2016 in the west cost of Canada and the USA. The epicenters of the earthquakes are indicated by coloured-stars whose size is proportional to the magnitude of the earthquake.

$82 \times 51 \mathrm{~mm}(300 \times 300 \mathrm{DPI})$ 


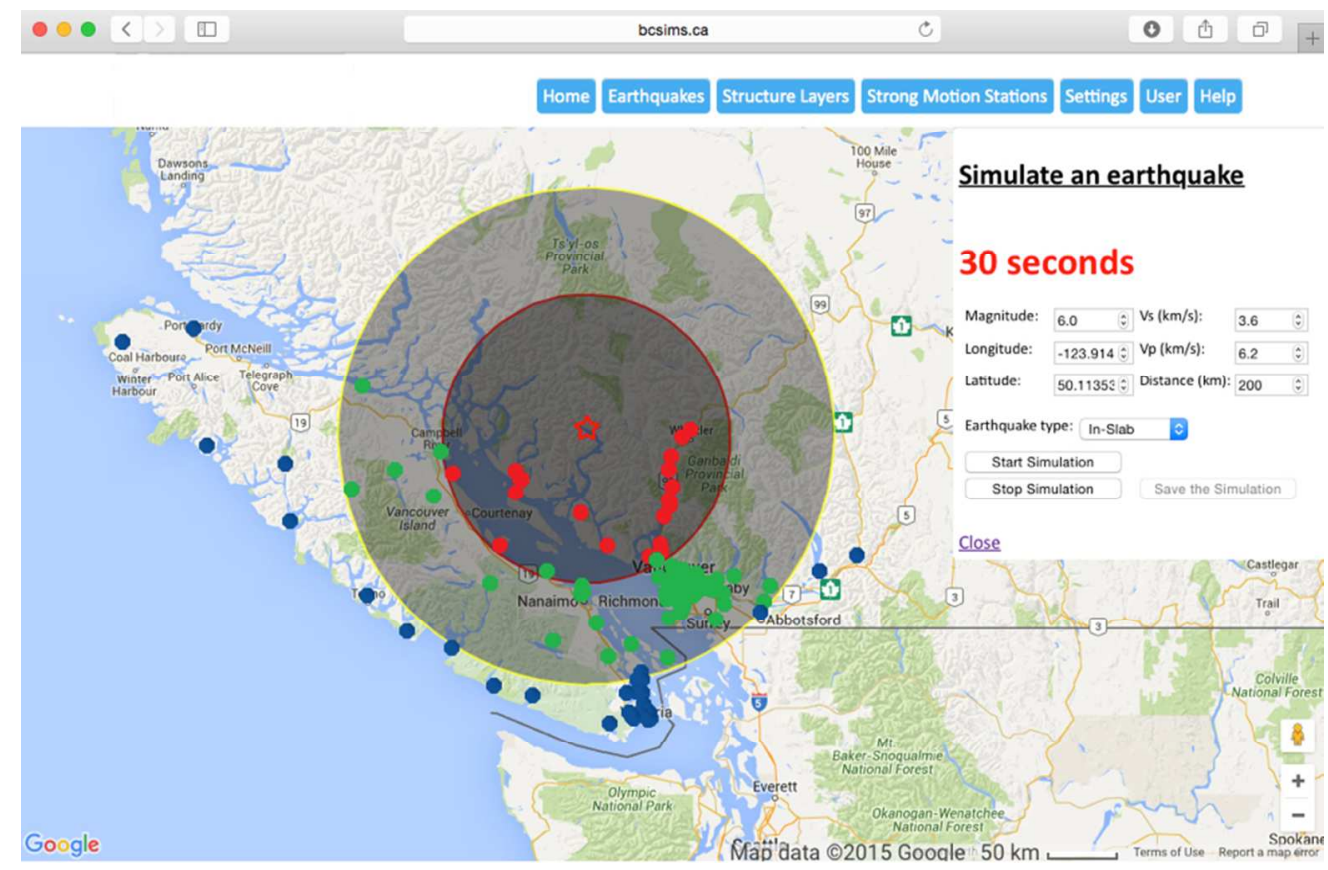

Earthquake simulation tool on the BCSIMS website enables users to simulate $\mathrm{p}$ - and $\mathrm{s}$ - waves of an earthquake. The two-growing yellow and red circles indicate the theoretical $p$ - and s-waves propagation. As these waves propagate, the colour of the SM stations will change from blue to green and red: the $\mathrm{p}$ - and $\mathrm{s}$ waves triggered the IA station. After the simulation is over, the final size and the colour of the IA stations indicate the expected PGA and kSI (Katayama et al. 1998).

$82 \times 53 \mathrm{~mm}(300 \times 300 \mathrm{DPI})$ 


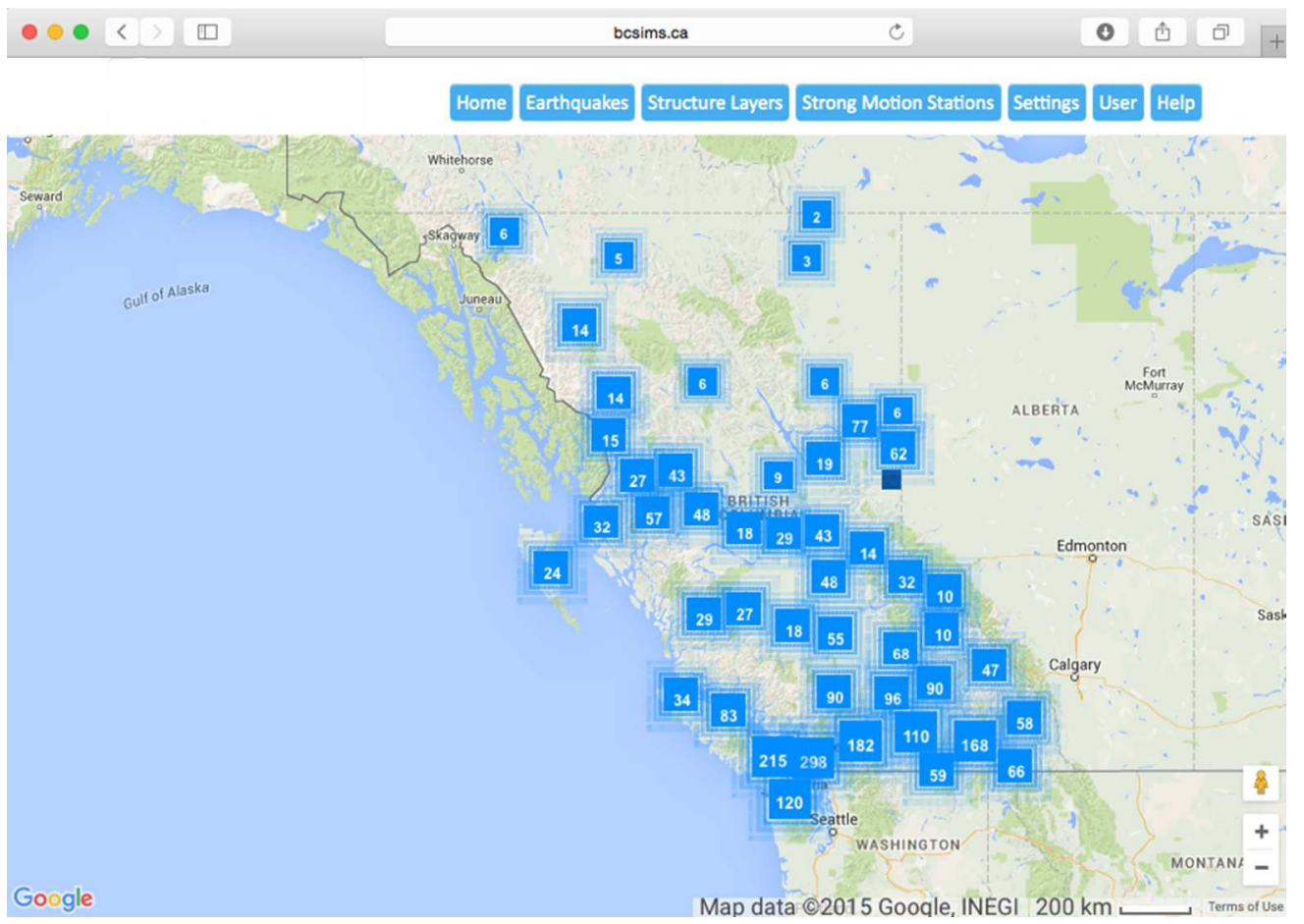

The locations of bridges owned by the Ministry of Transportation in British Columbia. The numbers in the square indicates number of bridges located under that square, and there are 2594 bridges in total and 14 of them have seismic SHM monitoring system installed (Table 1).

$82 \times 58 \mathrm{~mm}(300 \times 300$ DPI $)$ 


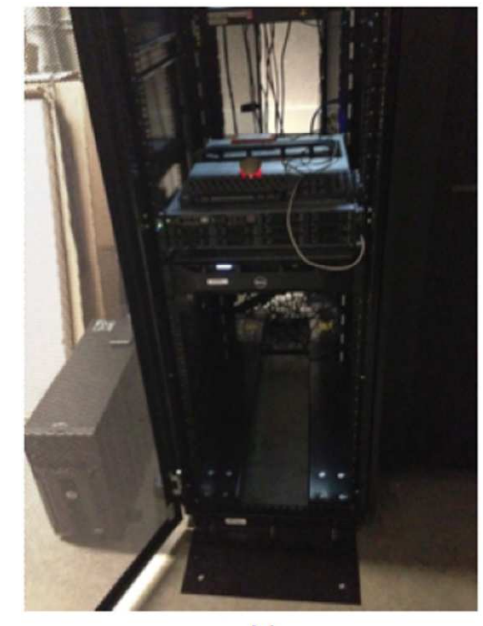

(a)

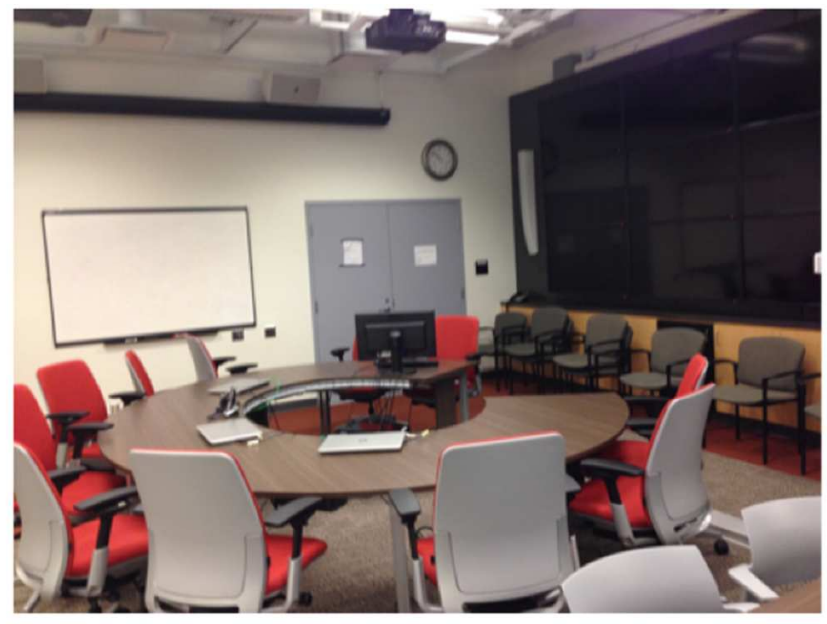

(b)

Monitoring control room at UBC, (a) the main server and data processing server in the control room, (b) the situation-room established in the control room.

$$
84 \times 42 \mathrm{~mm}(300 \times 300 \text { DPI })
$$

